\title{
Variabilidade na mensuração das medidas orofaciais
}

\author{
Variability of orofacial measures
}

\author{
Fernanda Veloso Rodrigues ${ }^{1}$, Fabiana Rodrigues Cerqueira Monção ${ }^{2}$, Michelle Barroso Rocha Moreira ${ }^{3}$, Andréa \\ Rodrigues Motta ${ }^{4}$
}

\begin{abstract}
RESUMO
Objetivo: Verificar o grau da variabilidade das medidas orofaciais, entre fonoaudiólogos especialistas em Motricidade Orofacial. Métodos: Estudo longitudinal prospectivo, tendo 30 fonoaudiólogos especialistas em Motricidade Orofacial realizado, em dois momentos distintos, com uso de paquímetro digital, a mensuração de dez medidas orofaciais de um sujeito padrão. Foram comparados os dados inter e intra-examinador por meio das medidas de tendência central, das medidas de dispersão e de testes de hipóteses, com nível de significância de 5\%. Resultados: Nove dos dez parâmetros investigados foram considerados como homogêneos na avaliação inter e intra-observadores. A abertura máxima da boca com língua na papila apresentou coeficientes de variação altos, indicando dados heterogêneos, apesar dos valores de correlação inter-observadores terem sido considerados semelhantes. Já na avaliação intraexaminador para este parâmetro foi evidenciada uma diferença significante $(\mathrm{p}=0,0384)$. Na análise dos dados, observou-se ainda diferenças próximas ao ponto de corte estabelecido no estudo, na mensuração intra-observador do terço médio da face $(\mathrm{p}=0,0711) \mathrm{e}$ da abertura máxima da boca ( $\mathrm{p}=0,0677)$. Dentre as dez variáveis analisadas, não foi observada diminuição do coeficiente de variação da $1^{\text {a }}$ para $2^{\mathrm{a}}$ mensuração apenas nos parâmetros terço médio da face e lábio superior. Conclusão: Não foi observada variabilidade nas mensurações orofaciais realizadas, exceto para o parâmetro abertura máxima da boca com língua na papila, indicando que dados produzidos por diferentes profissionais podem ser considerados parcialmente confiáveis.
\end{abstract}

Descritores: Antropometria; Face; Sistema estomatognático; Terapia miofuncional

\section{INTRODUÇÃO}

A Fonoaudiologia caracteriza os distúrbios miofuncionais orofaciais quando são diagnosticadas alterações posturais, morfológicas, de força e mobilidade dos órgãos fonoarticulatórios e, também, das funções estomatognáticas. Desta forma, a avaliação miofuncional deve ser completa e detalhada, relacionando cada dado obtido ${ }^{(1)}$.

$\mathrm{Na}$ prática fonoaudiológica, é de extrema importância avaliar os aspectos morfológicos das estruturas faciais devido à participação que as funções de mastigação, deglutição e respiração requerem destas estruturas, sendo a mensuração

Trabalho realizado no CEFAC - Saúde e Educação - Belo Horizonte (MG), Brasil.

(1) Especialista em Motricidade Orofacial , Fonoaudióloga da Prefeitura Municipal de Francisco Sá - Francisco Sá (MG), Brasil.

(2) Especialista em Motricidade Orofacial, Fonoaudióloga da Clínica SISO - Sete Lagoas (MG), Brasil.

(3) Especialista em Motricidade Orofacial, Fonoaudióloga da Clínica Habilitar Infantil - Uberlândia (MG), Brasil.

(4) Pós-graduanda em Distúrbios da Comunicação Humana pela Universidade Federal de São Paulo - UNIFESP - São Paulo (SP), Brasil; Professora Assistente do Departamento de Fonoaudiologia da Universidade Federal de Minas Gerais - UFMG - Belo Horizonte (MG), Brasil.

Endereço para correspondência: Fernanda Veloso Rodrigues. R. Coriolano Gonzaga, 1220, Major Prates, Montes Claros - MG, CEP 39403-215. E-mail: fefevrs@hotmail.com

Recebido em: 1/11/2007; Aceito em: 8/9/2008 das medidas faciais um dos aspectos morfológicos mais importante ${ }^{(2)}$. São descritos na literatura alguns procedimentos que podem ser utilizados por fonoaudiólogos na avaliação da morfologia orofacial ${ }^{(3-5)}$, tais como: a antroposcopia e a antropometria direta indireta. No que diz respeito às medidas antropométricas da região orofacial e aos seus padrões de normalidade, observa-se escassez na literatura nacional, sendo que por vezes na avaliação do sistema estomatognático, tais medidas são desconsideradas ${ }^{(6)}$. Entretanto, recentemente muitos estudos têm sido conduzidos no Brasil, buscando-se minimizar esta falta de padronização $0^{(1-2,6-6)}$.

A mensuração das estruturas orofaciais na avaliação fonoaudiológica contribui tanto para o estabelecimento da conduta terapêutica, como para o acompanhamento dos resultados da fonoterapia ${ }^{(8)}$. Dessa forma, torna-se fundamental investigar se os dados coletados pelos fonoaudiólogos apresentam confiabilidade.

Alguns estudos sobre reprodutibilidade em antropometria têm sido conduzidos ao longo do tempo ${ }^{(9-12)}$; entretanto, nenhum no campo fonoaudiológico. No que se refere aos aspectos orofaciais verifica-se que os pontos antropométricos por vezes refletem os pontos cefalométricos de cada indivíduo ${ }^{(13)}$. Todavia, algumas medidas realizadas a partir da cefalometria tendem a ser menores que as realizadas na face do indivíduo ${ }^{(14)}$. Cuidados especiais quanto à qualidade dos dados devem ser tomados, especialmente em estudo multicêntricos ${ }^{(15)}$. 
Assim, o objetivo da presente pesquisa foi verificar o grau da variabilidade das mensurações orofaciais entre fonoaudiólogos especialistas em Motricidade Orofacial.

\section{MÉTODOS}

Neste estudo longitudinal prospectivo, um total de 30 fonoaudiólogos, especialistas em Motricidade Orofacial, realizaram a mensuração das medidas orofaciais de um sujeito padrão. As avaliações foram realizadas na cidade de Belo Horizonte (MG), sendo que o sujeito padrão (uma das pesquisadoras) se deslocou até o local de trabalho de cada profissional.

Os critérios de seleção neste estudo foram: ser especialista em Motricidade Orofacial com prática clínica de pelo menos um ano; além de fazer uso do paquímetro rotineiramente.

Após a apresentação da pesquisa aos profissionais, os mesmos assinaram o termo de consentimento livre e esclarecido e foram solicitados a realizar com o mesmo paquímetro digital as seguintes mensurações ${ }^{(4,5)}$ : terço superior da face (tr-g); terço médio da face ( $\mathrm{g}$-sn); terço inferior da face (sn-gn); filtro labial (sn-ls); lábio superior (sn-sto); lábio inferior (sto-gn); distância comissura direita $\mathrm{x}$ canto do olho direito e a distância comissura esquerda x canto do olho esquerdo (ch-ex); bem como $^{(16)}$ abertura máxima da boca e abertura máxima da boca com língua na papila.

O sujeito padrão permaneceu sentado com os pés apoiados no chão, com a cabeça na posição habitual e lábios ocluídos ${ }^{(5)}$, uma vez que, em decorrência da relação entre o sistema estomatognático e o complexo crânio-cervical, a postura durante a mensuração pode alterar os resultados ${ }^{(17)}$. Entretanto, nenhuma outra orientação foi ministrada, uma vez que o direcionamento da conduta, como solicitar a realização da marcação dos pontos antropométricos ou estabelecer o número de medidas a cada dia, poderia interferir na rotina real praticada por cada profissional.

Para a realização desta pesquisa os materiais utilizados foram: protocolo de avaliação para anotação individual das medidas, luvas descartáveis, algodão, álcool etílico hidratado, detergente e paquímetro digital (Paquímetro Digital - 100.176 BL/Digimess Instrumentos de Precisão Ltda.). Ao final de cada avaliação foi realizada a higienização do instrumento. O período de cada avaliação para coleta das medidas foi de aproximadamente dez minutos.

Para cada parâmetro, uma pesquisadora fez a leitura do paquímetro, anotando os valores encontrados em um protocolo (Figura 1). Após um período mínimo de 30 dias, o mesmo procedimento foi repetido, sem que o profissional tivesse acesso às anotações do protocolo anterior.

Foram comparadas as medidas inter e intra-examinador. $\mathrm{O}$ banco de dados foi estruturado a partir do programa estatístico SPSS, tendo sido os dados analisados por meio das medidas de tendência central, das medidas de dispersão e de testes de hipóteses, considerando-se para tanto, um nível de significância de $5 \%(\mathrm{p}<0,05)$.

A análise da variabilidade em cada dia de mensuração (inter-examinador) foi realizada por meio das medidas de tendência central e dispersão. As análises das características da dispersão dos resultados foram baseadas no desvio padrão e no coeficiente de variação. O coeficiente de variação é uma

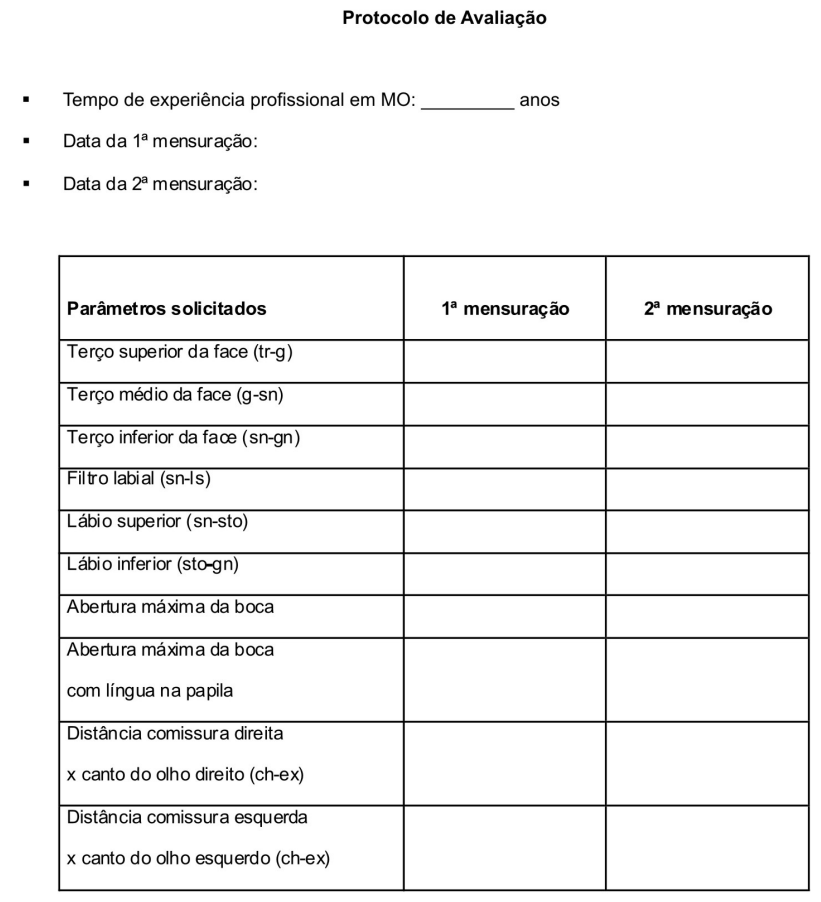

Figura 1. Protocolo de respostas empregado no estudo

medida adimensional e sua utilidade é fornecer uma medida para a homogeneidade do conjunto de dados. Um valor de coeficiente de variação menor ou igual a 0,25 geralmente indicará que o conjunto de dados é razoavelmente homogêneo. Uma classificação ${ }^{(18)}$ que pode ser utilizada é: baixo (inferior a 0,10$)$; médio (de 0,10 a 0,20$)$; alto de $(0,20$ a 0,30$)$; e muito alto (superior a 0,30 ).

Para a análise da variabilidade do $1^{\circ}$ para o $2^{\circ}$ dia de mensuração, foram considerados os dados inter-examinadores, empregando o teste de correlação de Pearson. Na análise de seus valores, considera-se a hipótese invertida, ou seja, a hipótese nula neste caso caracteriza ausência de correlação entre os dados.

Por fim, para a análise intra-examinador foi utilizado o teste de Wilcoxon, uma vez que todas as variáveis apresentaram distribuição não gaussiana, exceto no que se refere à distância canto do olho x comissura, sendo neste caso empregado o teste t-pareado.

Esse trabalho foi aprovado pelo Comitê de Ética em Pesquisa do CEFAC - Saúde e Educação sob o nº 31/06.

\section{RESULTADOS}

O tempo médio de experiência clínica em Motricidade Orofacial na amostra estudada foi de 4,4 anos. Cada fonoaudiólogo participante realizou dez medidas orofaciais em cada dia, totalizando 600 mensurações. Os dados gerais da $1^{\mathrm{a}}$ e $2^{\mathrm{a}}$ mensuração, assim como a análise estatística são descritas nas Tabelas de 1 a 4.

\section{DISCUSSÃO}

Com os resultados obtidos, foi possível verificar a variabilidade das medidas orofaciais realizadas por 30 fonoaudiólogos, 
Tabela 1. Dados referentes à mensuração dos terços da face

\begin{tabular}{|c|c|c|c|c|c|c|}
\hline \multirow[t]{2}{*}{ Análises } & \multicolumn{2}{|c|}{ Terço superior } & \multicolumn{2}{|c|}{ Terço médio } & \multicolumn{2}{|c|}{ Terço inferior } \\
\hline & $1^{\circ}$ dia & $2^{\circ} \mathrm{dia}$ & $1^{\circ} \mathrm{dia}$ & $2^{\circ} \mathrm{dia}$ & $1^{\circ} \mathrm{dia}$ & $2^{\circ}$ dia \\
\hline Média & $50,4 \mathrm{~mm}$ & $50,2 \mathrm{~mm}$ & $53,5 \mathrm{~mm}$ & $54,2 \mathrm{~mm}$ & $59,3 \mathrm{~mm}$ & $59,8 \mathrm{~mm}$ \\
\hline Mediana & $50 \mathrm{~mm}$ & $50 \mathrm{~mm}$ & $54 \mathrm{~mm}$ & $55 \mathrm{~mm}$ & $60 \mathrm{~mm}$ & $60 \mathrm{~mm}$ \\
\hline Limite superior & $54 \mathrm{~mm}$ & $54 \mathrm{~mm}$ & $57 \mathrm{~mm}$ & $58 \mathrm{~mm}$ & $63 \mathrm{~mm}$ & $65 \mathrm{~mm}$ \\
\hline Limite inferior & $46 \mathrm{~mm}$ & $46 \mathrm{~mm}$ & $47 \mathrm{~mm}$ & $46 \mathrm{~mm}$ & $54 \mathrm{~mm}$ & $54 \mathrm{~mm}$ \\
\hline Desvio padrão & $2,54 \mathrm{~mm}$ & $2,10 \mathrm{~mm}$ & $3,01 \mathrm{~mm}$ & $3,27 \mathrm{~mm}$ & $2,93 \mathrm{~mm}$ & $2,59 \mathrm{~mm}$ \\
\hline Coeficiente de variação & 0,050 & 0,042 & 0,056 & 0,060 & 0,049 & 0,043 \\
\hline Valor-p* & \multicolumn{2}{|c|}{0,0002} & \multicolumn{2}{|c|}{$<0,0001$} & \multicolumn{2}{|c|}{$<0,0001$} \\
\hline Valor $p^{* *}$ & \multicolumn{2}{|c|}{0,6091} & \multicolumn{2}{|c|}{0,0711} & \multicolumn{2}{|c|}{0,2059} \\
\hline
\end{tabular}

* Teste de Correlação de Pearson; ** Teste de Wilcoxon

Tabela 2. Dados referentes à mensuração dos lábios e filtro labial

\begin{tabular}{|c|c|c|c|c|c|c|}
\hline \multirow[t]{2}{*}{ Análises } & \multicolumn{2}{|c|}{ Lábio superior } & \multicolumn{2}{|c|}{ Lábio inferior } & \multicolumn{2}{|c|}{ Filtro labial } \\
\hline & $1^{\circ} \mathrm{dia}$ & $2^{\circ}$ dia & $1^{\circ} \mathrm{dia}$ & $2^{\circ}$ dia & $1^{\circ} \mathrm{dia}$ & $2^{\circ}$ dia \\
\hline Média & $19 \mathrm{~mm}$ & $19,5 \mathrm{~mm}$ & $43,3 \mathrm{~mm}$ & $44,2 \mathrm{~mm}$ & $12,9 \mathrm{~mm}$ & $13,2 \mathrm{~mm}$ \\
\hline Mediana & $19 \mathrm{~mm}$ & $19 \mathrm{~mm}$ & $45 \mathrm{~mm}$ & $47 \mathrm{~mm}$ & $13 \mathrm{~mm}$ & $13 \mathrm{~mm}$ \\
\hline Limite superior & $23 \mathrm{~mm}$ & $27 \mathrm{~mm}$ & $51 \mathrm{~mm}$ & $51 \mathrm{~mm}$ & $15 \mathrm{~mm}$ & $15 \mathrm{~mm}$ \\
\hline Limite inferior & $17 \mathrm{~mm}$ & $15 \mathrm{~mm}$ & $35 \mathrm{~mm}$ & $37 \mathrm{~mm}$ & $12 \mathrm{~mm}$ & $12 \mathrm{~mm}$ \\
\hline Desvio padrão & $1,67 \mathrm{~mm}$ & $2,56 \mathrm{~mm}$ & $5,09 \mathrm{~mm}$ & $4,66 \mathrm{~mm}$ & $1,08 \mathrm{~mm}$ & $1,02 \mathrm{~mm}$ \\
\hline Coeficiente de variação & 0,088 & 0,131 & 0,117 & 0,106 & 0,084 & 0,077 \\
\hline Valor-p* & \multicolumn{2}{|c|}{0,0002} & \multicolumn{2}{|c|}{$<0,0001$} & \multicolumn{2}{|c|}{0,0020} \\
\hline Valor $p^{\star \star}$ & \multicolumn{2}{|c|}{0,1683} & \multicolumn{2}{|c|}{0,1415} & \multicolumn{2}{|c|}{0,2809} \\
\hline
\end{tabular}

* Teste de Correlação de Pearson; ** Teste de Wilcoxon

Tabela 3. Dados referentes à mensuração da abertura da boca

\begin{tabular}{|c|c|c|c|c|}
\hline \multirow[t]{2}{*}{ Análises } & \multicolumn{2}{|c|}{$\begin{array}{c}\text { Abertura máxima da } \\
\text { boca }\end{array}$} & \multicolumn{2}{|c|}{$\begin{array}{c}\text { Abertura máxima da } \\
\text { boca com língua na } \\
\text { papila }\end{array}$} \\
\hline & $1^{\circ} \mathrm{dia}$ & $2^{\circ} \mathrm{dia}$ & $1^{\circ} \mathrm{dia}$ & $2^{\circ} \mathrm{dia}$ \\
\hline Média & $38,0 \mathrm{~mm}$ & $39,4 \mathrm{~mm}$ & $24,5 \mathrm{~mm}$ & $25,4 \mathrm{~mm}$ \\
\hline Mediana & $41 \mathrm{~mm}$ & $40 \mathrm{~mm}$ & $24 \mathrm{~mm}$ & $25 \mathrm{~mm}$ \\
\hline Limite superior & $46 \mathrm{~mm}$ & $48 \mathrm{~mm}$ & $41 \mathrm{~mm}$ & $43 \mathrm{~mm}$ \\
\hline Limite inferior & $27 \mathrm{~mm}$ & $26 \mathrm{~mm}$ & $16 \mathrm{~mm}$ & $16 \mathrm{~mm}$ \\
\hline Desvio padrão & $6,12 \mathrm{~mm}$ & $5,55 \mathrm{~mm}$ & $6,77 \mathrm{~mm}$ & $6,54 \mathrm{~mm}$ \\
\hline $\begin{array}{l}\text { Coeficiente de } \\
\text { variação }\end{array}$ & 0,161 & 0,141 & 0,276 & 0,258 \\
\hline Valor-p* & \multicolumn{2}{|c|}{$<0,0001$} & \multicolumn{2}{|c|}{$<0,0001$} \\
\hline Valor $p^{\star *}$ & \multicolumn{2}{|c|}{0,0677} & \multicolumn{2}{|c|}{0,0384} \\
\hline
\end{tabular}

* Teste de Correlação de Pearson; ** Teste de Wilcoxon

em um indivíduo padrão, em dois momentos diferentes. As mensurações utilizadas, bem como as orientações para realizálas encontram-se descritas em publicações nacionais na área fonoaudiológica ${ }^{(3-7)}$; entretanto, cabe ressaltar que nenhum participante marcou na pele do sujeito padrão os pontos antropométricos a serem utilizados como referência ou realizou as medidas mais de uma vez a cada dia.

Como estudos de reprodutibilidade em antropometria ${ }^{(9-12)}$
Tabela 4. Dados referentes à mensuração da distância da comissura labial e do canto do olho

\begin{tabular}{|c|c|c|c|c|}
\hline \multirow[t]{2}{*}{ Análises } & \multicolumn{2}{|c|}{$\begin{array}{c}\text { Distância do lado } \\
\text { direito }\end{array}$} & \multicolumn{2}{|c|}{$\begin{array}{l}\text { Distância do lado } \\
\text { esquerdo }\end{array}$} \\
\hline & $1^{\circ} \mathrm{dia}$ & $2^{\circ} \mathrm{dia}$ & $1^{\circ} \mathrm{dia}$ & $2^{\circ} \mathrm{dia}$ \\
\hline Média & $69 \mathrm{~mm}$ & $68,7 \mathrm{~mm}$ & $68,3 \mathrm{~mm}$ & $68,4 \mathrm{~mm}$ \\
\hline Mediana & $69 \mathrm{~mm}$ & 69 mm & $69 \mathrm{~mm}$ & $69 \mathrm{~mm}$ \\
\hline Limite superior & $76 \mathrm{~mm}$ & $75 \mathrm{~mm}$ & $75 \mathrm{~mm}$ & $75 \mathrm{~mm}$ \\
\hline Limite inferior & $61 \mathrm{~mm}$ & $61 \mathrm{~mm}$ & $61 \mathrm{~mm}$ & $61 \mathrm{~mm}$ \\
\hline Desvio padrão & $4,19 \mathrm{~mm}$ & $4,01 \mathrm{~mm}$ & $4,38 \mathrm{~mm}$ & $3,81 \mathrm{~mm}$ \\
\hline $\begin{array}{l}\text { Coeficiente de } \\
\text { variação }\end{array}$ & 0,061 & 0,058 & 0,064 & 0,056 \\
\hline Valor-p* & \multicolumn{2}{|c|}{$<0,0001$} & \multicolumn{2}{|c|}{$<0,0001$} \\
\hline Valor $p^{\star *}$ & \multicolumn{2}{|c|}{0,4086} & \multicolumn{2}{|c|}{0,7885} \\
\hline
\end{tabular}

* Teste de Correlação de Pearson; ** Teste T Pareado

empregam metodologias diversas, sendo estas distintas do presente trabalho, não foi possível comparar diretamente os dados encontrados.

Ao se analisar as medidas de tendência central e dispersão do terço superior da face no $1^{\circ}$ dia de mensuração, observou-se que o limite superior e inferior indicaram uma diferença de $8 \mathrm{~mm}$; a média e a mediana apresentaram valores próximos e o desvio padrão $(2,54 \mathrm{~mm})$ pode ser considerado baixo, 
assim como o coeficiente de variação $(5,0 \%)$. Desta forma, verificou-se pouca variação entre as medidas do terço superior, realizadas pelos 30 participantes no $1^{\circ}$ dia. $\mathrm{Na} 2^{\mathrm{a}}$ mensuração valores próximos ao do $1^{\circ}$ dia foram verificados, tendo o teste de correlação de Pearson confirmado que as medidas do $1^{\circ}$ dia podem ser consideradas semelhantes às do $2^{\circ}$ dia. $\mathrm{O}$ teste de Wilcoxon indicou não haver diferença entre cada examinador da $1^{\mathrm{a}}$ para $2^{\mathrm{a}}$ mensuração.

O mesmo raciocínio pode ser empregado para os terços: médio e inferior da face, observando-se, entretanto, que no terço médio a diferença entre o extremos encontrados nas medidas foi de $10 \mathrm{~mm}$ na $1^{\mathrm{a}}$ avaliação e $12 \mathrm{~mm}$ na $2^{\mathrm{a}}$ e que o desvio padrão mostrou-se o mais elevado entre os terços, assim como o coeficiente de variação. Por fim, ressalta-se o baixo valor- $p$ verificado no teste de Wilcoxon $(p=0,0711)$ que, apesar de não ser considerado significante, encontra-se muito próximo ao ponto de corte estabelecido $(\mathrm{p}<0,05)$. Assim, o terço médio indicou ser, ainda que reprodutível, a mensuração com maior variabilidade entre os três terços da face.

Quanto aos dados referentes ao lábio superior, analisando-se os valores encontrados no limite superior e inferior, verificou-se uma diferença de $6 \mathrm{~mm}$ na $1^{\mathrm{a}}$ mensuração e de $12 \mathrm{~mm}$ na $2^{\mathrm{a}}$ mensuração, caracterizando a maior diferença de limites entre dias, obtida nesse estudo. Observou-se neste parâmetro também a maior diferença entre os desvios padrões das duas mensurações. O coeficiente de variação do primeiro dia $(8,8 \%)$ caracterizou-se como baixo e do segundo, como médio $(13,1 \%)$. Desta forma, os dados da $1^{\mathrm{a}}$ mensuração podem ser considerados mais homogêneos do que na $2^{\mathrm{a}}$, apesar de, pelo teste de correlação empregado, sem diferença estatística, tanto na análise inter quanto intra-examinador.

Para o lábio inferior, os valores do coeficiente de variação foram classificados como médios, sendo de $11,7 \%$ no primeiro dia e $10,6 \%$ no segundo. Estes valores, por serem menores que $25 \%$, podem ser considerados como homogêneos ${ }^{(16)}$. Novamente, os dados mostraram-se semelhantes entre os dias, considerando-se todos os fonoaudiólogos em conjunto ou cada pesquisado individualmente.

$\mathrm{Na}$ análise das características dos resultados do filtro labial, pode-se constatar baixo coeficiente de variação e pequena amplitude $(3,0 \mathrm{~mm})$ nas duas mensurações, indicando pouca variação. Entre os dias também não foram observadas diferenças inter ou intra-observadores. Esta variável apresentou o menor desvio padrão do estudo, além da menor diferença entre desvios padrões, ressaltando-se que o filtro labial foi o menor parâmetro avaliado. Estes dados discordam de estudo ${ }^{(9)}$ que indicou ser a magnitude da medida um fator de interferência direta na reprodutibilidade e precisão dos dados. Outro trabalho $^{(10)}$ também ressalta o impacto negativo do tamanho reduzido da estrutura a ser mensurada, na avaliação da precisão e acurácia inter-observadores.

No que se refere aos dados de abertura máxima da boca, verificou-se grande diferença entre os valores máximos e mínimos obtidos em cada dia e coeficientes de variação classificados como médios ( $16,1 \%$ no primeiro dia e $14,1 \%$ no segundo). Não foram observadas diferenças entre o grupo como um todo, comparando-se os dias, mas na avaliação intra-examinador verificou-se valor muito próximo ao significante $(\mathrm{p}=0,0677)$.
Assim, apesar de dados homogêneos, a abertura máxima de boca mostrou-se como um parâmetro mais variável.

A única diferença significante do presente estudo foi verificada na análise dos dados de abertura máxima da boca com língua na papila. Observou-se, neste caso, as maiores diferenças entre os limites: máximo se mínimos, $25 \mathrm{~mm}$ no $1^{\circ}$ dia e $27 \mathrm{~mm}$ no $2^{\circ}$, além de coeficientes de variação classificados como altos (27,6\% e $25,8 \%$ respectivamente). Desta forma, verificou-se que os dados coletados nos dois dias são heterogêneos. Entretanto, os valores de correlação inter-observadores foram considerados como semelhantes; assim, a mesma variação presente na $1^{\mathrm{a}}$ medida, manteve-se na $2^{\text {a }}$. Já na avaliação intra-examinador o teste de Wilcoxon evidenciou diferença significante $(\mathrm{p}=0,0384)$ indicando que apesar de em grupo os fonoaudiólogos se comportarem de forma semelhante, as mensurações feitas por cada participante diferiram de um dia para o outro.

$\mathrm{Na}$ avaliação clínica, as medidas da abertura máxima da boca com e sem a língua na região da papila palatina são fundamentais, especialmente para verificação do impacto do frênulo lingual no sistema estomatognático ${ }^{(16)}$. Considerandose os resultados do presente estudo, os dados obtidos para um mesmo paciente com objetivo de reavaliação não se mostram confiáveis, mesmo sendo realizados pelo mesmo profissional. Entretanto, o maior impacto se refere às pesquisas, uma vez que dados de publicações sobre o parâmetro acima descrito podem não apresentar confiabilidade, caso as medidas sejam tomadas em momentos diferentes. Este fato pode interferir negativamente na comparação de informações, comprometendo o estabelecimento de parâmetros norteadores da prática fonoaudiológica. Assim, cabe aos fonoaudiólogos atentarem para este aspecto, buscando melhorar a qualidade das informações produzidas.

Por fim, quanto aos dados da distância entre comissura labial e canto externo do olho, do lado direito, apesar de uma diferença entre os limites superiores e inferiores nos dois dias, os coeficientes de variação apresentaram-se baixos, $6,1 \%$ e $5,8 \%$, respectivamente. O teste de correlação de Pearson não indicou variação entre os dias, assim como o Teste t-pareado na avaliação intra-observador. Na avaliação do lado esquerdo, os dados apresentaram-se de forma bastante semelhante.

Algumas considerações podem ser realizadas, apesar de nove dos dez parâmetros investigados terem sido considerados como homogêneos na avaliação inter e intra-observadores. $\mathrm{Na}$ comparação inter-observadores, entre os dois dias de mensuração, observou-se no lábio superior diferença de classificação dos coeficientes de variação (baixo e médio respectivamente), entretanto, nenhuma diferença foi observada por meio do teste de correlação de Pearson. Quanto aos aspectos intraobservador, foram obtidos valores próximos aos significantes, na mensuração do terço médio da face e da abertura máxima da boca.

Apesar de estudos ${ }^{(9-10)}$ fazerem referência aos erros inerentes à antropometria, especialmente nos casos em que os pontos de referência são estimados e não visualizados diretamente, no presente estudo, o mais baixo coeficiente de variação foi verificado na avaliação do terço superior da face, que envolve determinação de ponto por estimativa. 
Dentre as dez variáveis analisadas, em apenas duas não foi observada diminuição do coeficiente de variação da $1^{\mathrm{a}}$ para $2^{\mathrm{a}}$ mensuração, a saber: terço médio da face e lábio superior. Estudo anterior ${ }^{(10)}$ verificou melhora da reprodutibilidade no $2^{\circ}$ dia; entretanto, foi realizada nova calibração entre os examinadores, entre as duas mensurações. Apesar deste aspecto não ter feito parte da metodologia empregada na presente pesquisa, acredita-se que os profissionais possam ter buscado sanar possíveis dúvidas que tenham surgido no momento da avaliação. Sabe-se que tanto o conhecimento sobre o protocolo empregado, quanto o treinamento e experiência clínica influenciam diretamente na reprodutibilidade das medidas antropométricas $^{(11)}$.

É essencial que o profissional que busca utilizar as medidas antropométricas orofaciais na rotina clínica tenha conhecimento dos pontos de referência e habilidade para manusear o paquímetro $^{(5)}$. Apesar de ter sido verificado na amostra estudada que não ocorreu marcação dos pontos antropométricos ou repetição das medidas para realização da média, os fonoaudiólogos especialistas em Motricidade Orofacial produzem dados consistentes, fato fundamental quando se compara dados de profissionais distintos.

Ressalta-se que, além de registrar os dados de mensuração dos aspectos orofaciais, é de suma importância que o profissional relacione e interprete estes. Caberá, ainda ao fonoaudiólogo descrever as possibilidades e limites da fonoterapia ${ }^{(19)}$. Portanto, é fundamental também o conhecimento dos padrões de normalidade existentes ${ }^{(4,5)}$ para que seja possível compreender os dados obtidos.

Em decorrência do reduzido tamanho da amostra e, considerando-se que de uma maneira geral, os fonoaudiólogos avaliaram de forma semelhante o indivíduo padrão do estudo, novos estudos devem ser conduzidos, buscando-se a confirmação dos dados, uma vez que, contrariamente ao observado no presente trabalho, a literatura aponta para erros maiores inter-observadores do que intra-observadores nas avaliações antropométricas ${ }^{(10)}$.

\section{CONCLUSÃO}

Não foi observada variabilidade nas mensurações orofaciais realizadas por fonoaudiólogos especialistas em Motricidade Orofacial, exceto para o parâmetro abertura máxima da boca com língua na papila, indicando que dados produzidos por diferentes profissionais podem ser considerados parcialmente confiáveis.

\begin{abstract}
Purpose: To verify the degree of variability of orofacial measures, among speech pathologists who are specialists in orofacial myology. Methods: A prospective study was carried out, where 30 speech pathologists who are specialists in orofacial myology measured, in two different moments, with the use of a digital sliding caliper, ten orofacial measures of a standard subject. Withinand between-examiner data were compared using measures of central trend and dispersion, and hypothesis testing, with the level of significance of 5\%. Results: Nine out of the ten parameters investigated were considered as homogeneous in both within- and between-observers analyses. The maximum mouth opening with the tongue in the papilla presented high coefficients of variation, indicating heterogeneous data, although between-observers values were considered similar. In the within-examiner analysis for this parameter, a significant difference was evidenced $(\mathrm{p}=0.0384)$. In the data analysis, it was also observed differences next to the cut point established in the study, in the within-observer mensuration of the midfacial height $(p=0.0711)$ and the maximum mouth opening ( $p=0.0677$ ). Among the ten analyzed variables, it was not observed decrease in the coefficient of variation from the first to the second mensuration only for the parameters midfacial height and superior lip. Conclusion: It was not observed variability in the orofacial mensuration carried out in this study, except for the parameter maximum mouth opening with the tongue in the incisor papilla, indicating that data produced by different professionals can be considered partially trustworthy.
\end{abstract}

Keywords: Anthropometry; Face; Stomatognathic system; Myofunctional therapy

\title{
REFERÊNCIAS
}

1. Cattoni DM, Fernandes FD, Andrade CR, Latorre MR. Estudo sobre as medidas faciais em crianças: correlações com alteração de mordida e uso de aparelho ortodôntico. Rev Soc Bras Fonoaudiol. 2005;10(1):1-6.

2. Cattoni DM, Fernandes FD. Medidas e proporções faciais em crianças: contribuições para a avaliação miofuncional orofacial. Pró-Fono. 2004;10(1): 7-18.

3. Silva HJ, Cunha DA. Considerações sobre o uso do paquímetro em motricidade oral. Rev Fonoaudiol Brasil. 2003;2(4):59-66.

4. Cattoni DM. Avaliação quantitativa das estruturas orofaciais. In: Krakauer LH, Di Francesco RC, Marchesan IQ. Respiração oral. Abordagem interdisciplinar. São José dos Campos: Pulso; 2003. p. 8188.
5. Cattoni DM. O uso do paquímetro na motricidade orofacial. Procedimentos de avaliação. Barueri: Pró-Fono; 2006.

6. Parro FM, Toledo MR, Gomes ICD, Marchesan IQ. Diferenças antropométricas entre mulheres brancas e negras após crescimento puberal. Rev CEFAC. 2005;7(4):459-65.

7. Cattoni DM, Fernandes FDM, Marchesan IQ, Latorre MRDO Medidas antropométricas faciais em crianças segundo os períodos da dentição mista. Rev CEFAC. 2003;5(1):21-9.

8. Jardini RSR. Avaliação facial a partir da relação eletromiográfica e antropométrica do músculo bucinador. Rev Soc Bras Fonoaudiol. 2005;10(3):161-7.

9. Ward RE, Jamison PL. Measurement precision and reliability in 
craniofacial anthropometry: implications and suggestions for clinical applications. J Craniofac Genet Dev Biol. 1991;11(3):156-64.

10. Kouchi M, Mochimaru M, Tsuzuki K, Yokoi T. Interobserver errors in anthropometry. J Hum Ergol (Tokyo). 1999;28(1-2):15-24.

11. Vegelin AL, Brukx LJ, Waelkens JJ, Van den Broeck J. Influence of knowledge, training and experience of observers on the reliability of anthropometric measurements in children. Ann Hum Biol. 2003;30(1):65-79

12. Mommaerts MY, Moerenhout BA. Reliability of clinical measurements used in the determination of facial indices. J Craniomaxillofac Surg. 2008; 36(5):279-84

13. Budai M, Farkas LG, Tompson B, Katic M, Forrest CR. Relation between anthropometric and cephalometric measurements and proportions of the face of healthy young white adult men and women. J Craniofac Surg. 2003;14(2):154-61.
14. Farkas LG, Tompson BD, Katic MJ, Forrest CR. Differences between direct (anthropometric) and indirect (cephalometric) measurements of the skull. J Craniofac Surg. 2002;13(1):105-8.

15. Farkas LG, Katic MJ, Forrest CR, Alt KW, Bagic I, Baltadjiev G, et al. International anthropometric study of facial morphology in various ethnic groups/races. J Craniofac Surg. 2005;16(4):615-46.

16. Marchesan IQ. Frênulo lingual: proposta de avaliação quantitativa. Rev CEFAC. 2004;6(3):288-93.

17. Villanueva P, Valenzuela S, Santander H, Zúñiga C, Ravera MJ, Miralles $R$. Efecto de la postura de cabeza en mediciones de la via aérea. Rev CEFAC. 2004;6(1):44-8.

18. Soares JF, Siqueira AL. Introdução à estatística médica. 2a ed. Belo Horizonte: Coopmed; 2002.

19. Costa TLS, Silva, HJ, Cunha DA. Análise quanlitativa inter-observadores e avaliação morfométrica do palato duro. Rev CEFAC. 2005;7(3):32635 . 